\title{
A Hybrid Convolutional Neural Network-Support Vector Machine for X-ray Computed Tomography Images on Cancer
}

\author{
Jane Eva Aurelia*, Zuherman Rustam \\ Department of Mathematics, University of Indonesia, Depok, Jakarta, Indonesia
}



\section{Introduction}

Cancer is a malignant disease due to the uncoordinated growth of abnormal cells in body tissues [1], as one of the health problems with the most deaths throughout the world, including Indonesia. The World Health Organization (WHO) states that cancer as the main causes of death inflicts around $13 \%$ of all causes of death throughout the world [2]. In 2007, there were an estimated of 7.9 million cancer deaths in the world, that each year about 12 million people of cancer sufferers conduce 7.6 million of them die from cancer worldwide [3]. If not restrained, by 2030 , there will be 26 million cancer patients and 17 million cancer deaths [3]. Ironically, this event will happen more quickly in poor and developing countries like Indonesia. Based on RISKESDAS data, the proportion of cancer in Indonesia denoted an increase of 1.79 per 1000 population in 2018 from 2013 by 1.4 per 100 population [4]. The most common types of cancer that causes death every year are lung cancer with 1.4 million deaths/year followed by 653,000 deaths/year of liver cancer and 548,000 deaths/year of breast cancer [5]. Those types of cancers are different between men and women, where for men are lung and liver as the most common type, while lung and breast are for women.

Lung cancer is the most common disease caused death that occurs when abnormal cells grow in lung tissue [6]. Lung cancer causes men to die more often than other cancers, as ranked six out of 10 main causes of death in Indonesia, where dominated with cigarette smoke as the lead elements. Surgery of thoracic is one of the primary solutions [7], yet still has many perils and ravels that can induce to death. Furthermore, including Indonesia, liver cancer is also big res throughout the world. If a person is exposed to chronic hepatitis, it can progress to liver cirrhosis and eventually liver cancer in $20-30 \%$ of cases [8]. One of the preventions is the treatment of chronic hepatitis which reduces liver inflammation by eliminating or suppressing the replication of the causative virus [9], but until now there is no optimal therapy. Finally, breast cancer is a disease where the malignant cells are in the breast tissue [10]. This cancer is the most common cancer suffered by women in developed countries, including Indonesia, which can have problems in the psychological aspect. The incidence of breast cancer continues to increase 
and until now there have been no data that are sure to be the main cause of breast tumors or cancer [11].

Various healing approaches have been developed but the effects of cancer treatment have a negative impact on patients. Efforts to prevent and control cancer are being intensively carried out by the government, as for early detection and comprehensive management have been developed and aimed at people's risk. In the past decade, research on various prevention efforts is being carried out as focusing on better healing effectiveness. One of the focuses of our researchers is machine learning. The proposed method used is a hybrid convolutional neural network (CNN)support vector machine (SVM) as many researchers have been applied separately. Some of the previous studies among these models are brain tumors using CNN [12], coronavirus disease 2019 using CNN [13], hepatitis using SVM [14], and Alzheimer using SVM [15]. The purpose of writing this research is to find out if machine learning technique can be used in the prevention and treatment of cancer. The results aim to prevent and control cancer with the detection of cancers' type before the unwanted side effects. This research contains of the backgrounds of cancer at first section, followed by data materials and methods in the second section, along with the proposed method in the third section, results and analysis in the fourth section, finally ends with discussion to conclusion.

\section{Materials and Methods}

This section part explains the dataset used, along with the theoretical basis of hybrid CNN-SVM method and the evaluated model performance used.

\section{Data}

X-rays are an electromagnetic waves radiation that produces pictures of your inside body parts in diverse shades of black and white [16]. This black and white image is produced because the body's tissues absorb different amounts of radiation, where X-ray examination aims to help doctors diagnose and monitor several body conditions as cancer [16]. The data materials used to perform in this research consist of X-ray computed tomography (CT) images taken from someone affected by cancer in 2019-2021 of time range, obtained from cancer imaging archive. A total observation of $900 \mathrm{X}$-ray CT images that were mixed between normal conditions and those who had cancer cells, which consists of 226 , 241, 234, and 199 data for lung, liver, breast, and noncancer images, respectively. Figure 1 is an example of the image data materials used in this research.
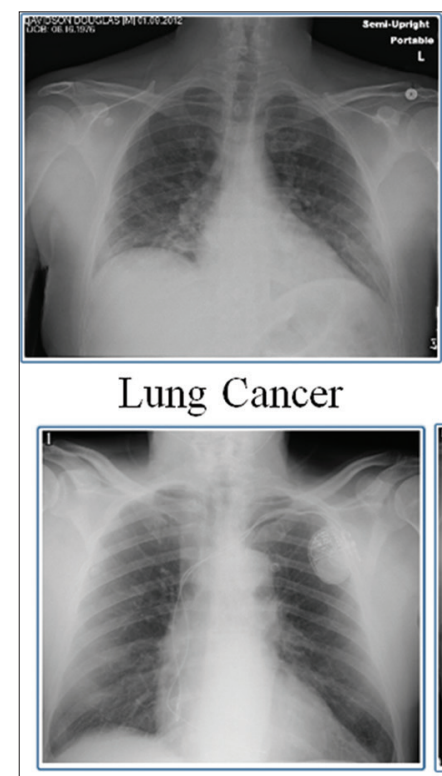

Breast Cancer

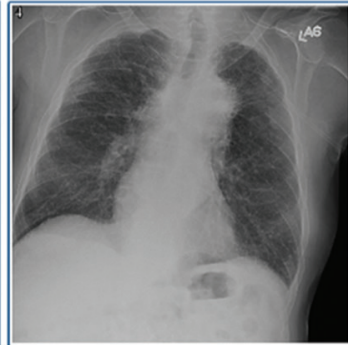

Liver Cancer

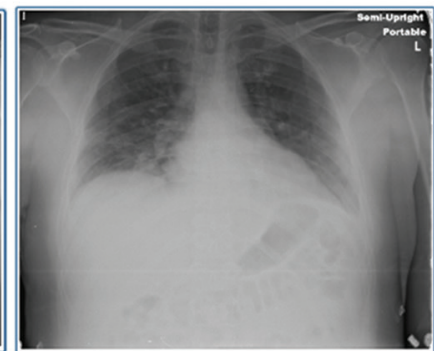

Normal
Figure 1: X-ray computed tomography image data materials used (Source: cancerimagingarchive.net)

\section{CNNs}

CNNs as one of machine learning methods of deep neural networks is a cultivation of multilayer perceptron (MLP) which designed to produce or process data from two dimensions that have a network tier and many applications carried out in the image [17]. CNN consists of learning stage utilizing backpropagation and feedforward for classification. The working principle of CNN is one of the further developments of MLP with more dimensions [18], where each neuron of CNN is presented in two dimensions that not the same as MLP that only has one dimension. The data propagated into CNN are two dimensions data, therefore, the calculations are carried out linearly and using different weight parameters on CNN. Linear calculations in the CNN method use convolution calculations, with weights that are no longer just one dimension, but are already in the form of four dimensions which are a collection of various convolution kernels [19]. The following figure is an overview of the steps used in the process using the CNN method, as shown in Figure 2 [20].

Similar as neural networks, CNN has certain hidden layers of an input that has a single vector, where inside the input is a digital image that made to a single vector and inside the hidden layers is divers' neurons that have four mapping features [21]. This research used three main types of layers to build CNN Architectures of Convolutional, Pooling, and Fully Connected Layer. These layers will stack to form of Mobile Net V2 Architecture with SVM classification on output layer as the last layer which connected to previous hidden layer that presented into class classification, where SVMs method is used in this research. 




Figure 2: Convolutional neural networks architecture (Source: Applied Sciences Journal)

\section{SVMs}

SVMs are a hypothetical space in the form of linear functions feature that has high dimensions and trained algorithm based on optimization theory [22]. SVM performs a technique to find a separator function that can separate two data sets from two different classes. SVM was first introduced by Vapnik in 1992 as a series of several superior concepts in the field of pattern recognition when it was presented at the Annual Workshop on Computational Learning Theory [23]. This classification method is developed and applied as rooted in statistical learning theory whose results are promising to provide better results. Based on its characteristics, the SVM method is divided into two [24], linear SVM that separated linearly of two classes on the hyperplane with soft margins and non-linear SVM that applying kernel trick function to highdimensional space. Figure 3 is an example of illustration of SVM method finds the best hyperplane [25].

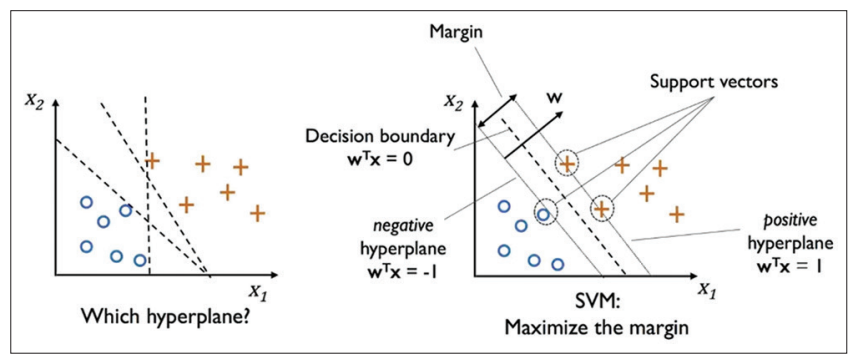

Figure 3: Support vector machines find the best hyperplane (Source: Python Machine Learning eBook)

The action of separating the two classes with a hyperplane is identified the right hyperplane, classifies the two classes, and then finds the hyperplane to separate to classes [26]. The hyperplane equation is shown in Equation (1) where $w$ is the weight and $b$ is the bias value. $w . x+b=0$

Using quadratic programming as its basic formulation as shown in Equation (2), with the constraints in Equation (3), Lagrange multiplier method also used as a technique in optimization problems with certain constraints as shown in Equation (4). $\min \left(\frac{1}{2}\|w\|^{2}\right)$

$y i(w . x i+b) \geq 1, \forall i=1, \ldots, N$

$$
\begin{gathered}
L(w, b, \alpha)=\frac{1}{2}\|w\|^{2}-\sum_{i=1}^{N} \alpha i(y i((w . x i+b)-1)), \\
i=1,2, \ldots, N
\end{gathered}
$$

Then, the values of $w$ and $b$ that obtained in Equations (5) and (6) are substituted to the function $f(x)$ in Equation (7).

$w=\sum_{i=1}^{N} \alpha i y i x i$

$b=\frac{1}{N s} \sum_{i \in S}\left(y_{i}-\sum_{j \in S} \alpha_{j} y_{j} x_{j}\right)$

$f(x)=\operatorname{sign}(w \cdot x+b)$

SVM regression as SVM for prediction is consisting of linear and nonlinear functions with primal and dual formulas [27], whose known as nonparametric technique because it relies on kernel functions. Generated by the transition process with SVM whose very dependent on the kernel function and the parameters used [27], types of kernels used in this research are Gaussian radial basis function (RBF) as described in Equation (8) below.

$K\left(x_{i}, x_{j}\right)=\exp \left(-\frac{x_{i}-x_{j}^{2}}{\sigma^{2}}\right)$ 


\section{Evaluated model performance}

As one of the evaluated model performances, accuracy is a main parameter for observing the classification successes [28], where the greater the accuracy, then the better the classifier's performance. On determining the classifier, refers to the percentage of correct answers in the testing stage, confusion matrix is used for accurate measures, as shown in Table 1 [28].

Table 1: Confusion matrix

\begin{tabular}{lll}
\hline Actual & Prediction & \\
\cline { 2 - 3 } & Positive & Negative \\
\hline Positive & $\mathrm{T}_{\mathrm{P}}$ & $\mathrm{F}_{\mathrm{N}}$ \\
Negative & $\mathrm{F}_{\mathrm{P}}$ & $\mathrm{T}_{\mathrm{N}}$ \\
\hline
\end{tabular}

Furthermore, the formula of accuracy, precision, and sensitivity as benchmark of the evaluated model performance is written in Equations (9), (10), and (11).

Accuracy $=\frac{T_{p}+T_{N}}{T_{P}+T_{N}+F_{P}+F_{N}}$

Precision $=\frac{T_{p}}{T_{P}+F_{P}}$

Sensitivity $=\frac{T_{p}}{T_{P}+F_{N}}$

Where:

- $\quad$ True positive $\left(T_{p}\right)$ is the number of samples suffering from cancer and correctly classified

- $\quad$ False positive $\left(F_{\mathrm{p}}\right)$ is the number of non-cancer individuals misclassified as cancer

- $\quad$ False negative $\left(F_{N}\right)$ is the number of samples of patients with cancer and incorrectly classified

- $\quad$ True negative $\left(\mathrm{T}_{\mathrm{N}}\right)$ is the number of non-cancer individuals that correctly classified.

\section{The Proposed Method}

The combining model of hybrid convolution neural network-SVM is applied to TensorFlow as a package framework that supports machine in the preparing of image data. The X-ray CT image data are classified into two classes, where the separation of image data using hold-out validation method that carried out in two parts with $10 \%$ up to $90 \%$ testing data. These different percentages were carried out to determine which testing data value of the model can use to maximize the classification. In convolutional part, binary cross-entropy and Adam (adaptive momentum) were used as the loss function and the optimizer of CNN model, respectively, along with batch of 50 and 1000 epochs. Furthermore, for classification part, Gaussian RBF was used as the kernel function of SVM model with parameter gamma equals 5. The evaluated model performance was accuracy, precision, sensitivity, and running time. In addition, the proposed method flowchart was formed in Figure 4 and the summary of CNN architecture used on hybrid CNN-SVM is shown in Figure 5 as the number of layers, parameters, and output shape included.

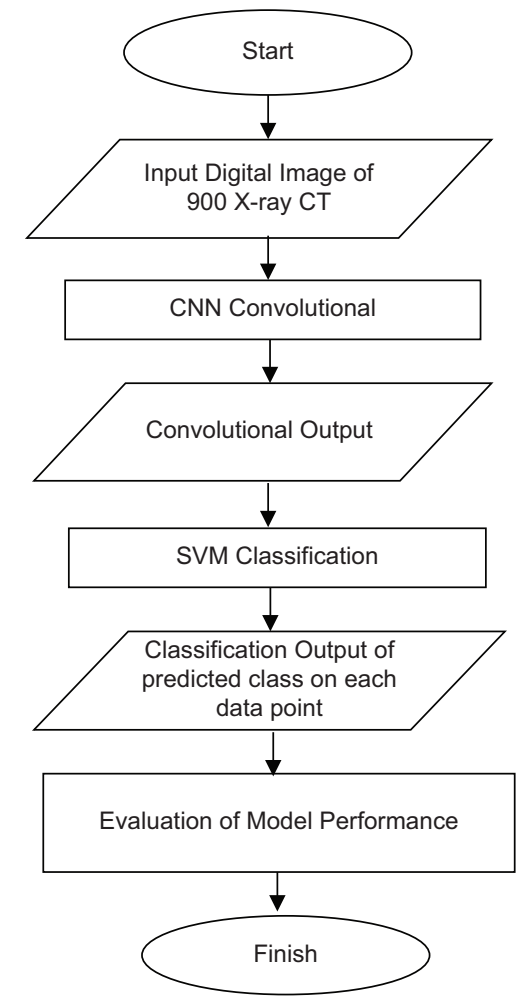

Figure 4: Flowchart of proposed method

Figure 4 shows the proposed method flowchart start with 900 X-ray CT images, with size of $300 \times 300$ pixels that examined into $\mathrm{CNN}$ architecture in Figure 4. Forwarded to bunch of CNN layers, the output vector will divide into two datasets as training and testing using hold-out validation method. The model in this research used SVM, then validate the model with testing set. Finally, based on the predicted model, the predicted class of every data point will be evaluated so could determine the best model performance.

Figure 5 shows the summary of $\mathrm{CNN}$ architecture used on hybrid CNN-SVM with input values of $\mathrm{n} \times 300 \times 1$. At the convolutional operation stage, four convolutional layers are used with first layer of $32-3 \times 3$ filters as the value of 32 means the number of filters used in the $3 \times 3$ matrices, followed by second layer of $64-3 \times 3$ filters, third layer of $96-3 \times 3$ filters, and fourth layer of $128-3 \times 3$ filters. By reducing the size of feature map's or dimension obtained using pooling layers, this model used measurement $2 \times 2$ of max pooling which was placed after all the convolutional layers. Due to the pooling layer's used, the largest value was obtained that create a new feature map. Using two strides, the filters on the layers of the previous matrix are shifts from left to right horizontally by two pixels before moving to the 


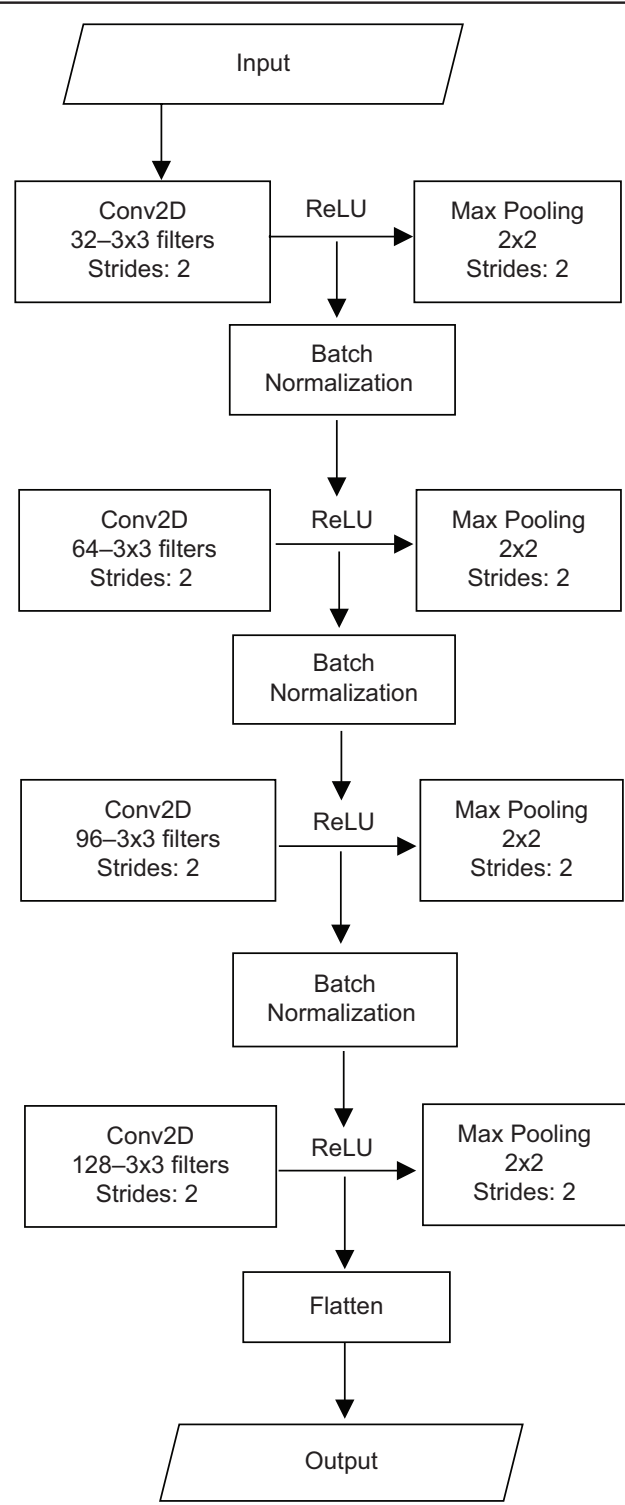

Figure 5: Summary of convolutional neural networks architecture

bottom leftmost vertically by two pixels. The result from most recent feature map was reconstructed by flattening where turning it into a one-dimensional array then finally the output.

\section{Results and Analysis}

The proceeds of the proposed method execution of hybrid CNN-SVM on X-ray CT images dataset are presented. The model's performance is tested to determine the evaluation of accuracy, precision, and sensitivity of prediction model on every testing data proportion implemented based on confusion matrix, along with their running time. The total observation of 900 X-ray CT images that contain of mixture conditions among normal and cancer sufferers of lung, liver, also breast, the results of the model's performance of Hybrid CNN-SVM with kernel functions of Gaussian RBF equals 5 parameters of gamma are summarized in Tables 2-4.
Table 2 shows that the model's performance of hybrid CNN-SVM with dataset of lung cancer is a proper and competent model, with average of running time of $\pm 0.6802 \mathrm{~s}$. At average of $86.95 \%$, the highest

Table 2: Hybrid CNN-SVM model's performance on cancer of lung

\begin{tabular}{llll}
\hline Testing data (\%) & Accuracy $(\%)$ & Precision $(\%)$ & Sensitivity $(\%)$ \\
\hline 10 & 99 & 98 & 99 \\
20 & 100 & 99 & 100 \\
30 & 98.03 & 98 & 98 \\
40 & 98.52 & 98 & 99 \\
50 & 89.41 & 88 & 98 \\
60 & 99.01 & 99 & 99 \\
70 & 99.15 & 99 & 99 \\
80 & 91.04 & 89 & 100 \\
90 & 95.42 & 95 & 98 \\
Average & 86.95 & 86.3 & 89 \\
\hline
\end{tabular}

accuracy result is $100 \%$ at $20 \%$ testing data, with the lowest accuracy result of $89.41 \%$ at $50 \%$ testing data. Moreover, with average of $86.3 \%$, the highest precision result is $99 \%$ at $20 \%, 60 \%$, and $70 \%$ testing data, as the lowest is $88 \%$ at $50 \%$ testing data. Finally, for the sensitivity, with average of $89 \%$, the highest and the lowest value resulted were $100 \%$ at $20 \%$ and $80 \%$ testing data and $98 \%$ at $30 \%, 50 \%$, and $90 \%$ testing data, respectively.

Table 3: Hybrid CNN-SVM model's performance on cancer of liver

\begin{tabular}{llll}
\hline Testing data (\%) & Accuracy $(\%)$ & Precision $(\%)$ & Sensitivity $(\%)$ \\
\hline 10 & 98.23 & 97 & 98 \\
20 & 99.17 & 98 & 100 \\
30 & 90.39 & 90 & 98 \\
40 & 90.88 & 90 & 98 \\
50 & 84.11 & 84 & 89 \\
60 & 92.35 & 91 & 90 \\
70 & 84.78 & 84 & 89 \\
80 & 91.61 & 90 & 90 \\
90 & 81.89 & 82 & 88 \\
Average & 81.34 & 80.6 & 84 \\
\hline
\end{tabular}

Table 3 shows that the model's performance of hybrid CNN-SVM with dataset of liver cancer is a proper and competent model, with average of running time of $\pm 0.7204 \mathrm{~s}$. At average of $91.34 \%$, the highest accuracy result is $99.17 \%$ at $20 \%$ testing data, with the lowest accuracy result of $81.89 \%$ at $90 \%$ testing data. Moreover, with average of $80.6 \%$, the highest precision result is $98 \%$ at $20 \%$ testing data, as the lowest is $82 \%$ at $90 \%$ testing data. Finally, for the sensitivity, with average of $84 \%$, the highest and the lowest values resulted were $100 \%$ at $20 \%$ testing data and $88 \%$ at $90 \%$ testing data, respectively.

Table 4: Hybrid CNN-SVM model's performance on cancer of breast

\begin{tabular}{llll}
\hline Testing data (\%) & Accuracy (\%) & Precision (\%) & Sensitivity (\%) \\
\hline 10 & 96.47 & 96 & 100 \\
20 & 98.35 & 99 & 100 \\
30 & 97.39 & 99 & 100 \\
40 & 95 & 95 & 98 \\
50 & 94.11 & 94 & 98 \\
60 & 94.5 & 95 & 98 \\
70 & 92.26 & 92 & 90 \\
80 & 92.79 & 93 & 90 \\
90 & 91.89 & 92 & 89 \\
Average & 85.27 & 85.5 & 86.3 \\
\hline
\end{tabular}

Table 4 shows that the model's performance of hybrid CNN-SVM with dataset of breast cancer is a proper and competent model, with average of running time of $\pm 0.6853 \mathrm{~s}$. At average of $85.27 \%$, the highest accuracy result is $98.35 \%$ at $20 \%$ testing data, with the 
lowest accuracy result of $91.89 \%$ at $90 \%$ testing data. Moreover, with average of $85.5 \%$, the highest precision result is $99 \%$ at $20 \%$ and $30 \%$ testing data, as the lowest is $92 \%$ at $90 \%$ testing data. Finally, for the sensitivity, with average of $86.3 \%$, the highest and the lowest values resulted were $100 \%$ at $10 \%-30 \%$ testing data and $89 \%$ at $90 \%$ testing data, respectively. The authors also keep track of the comparison of accuracy generated between hybrid CNN-SVM programs in classifying all cancer data based on X-ray CT images. The comparison of hybrid CNN-SVM accuracy is shown in Table 5 and Figure 6.

Table 5: Comparison of hybrid CNN-SVM accuracy's performance on cancer dataset

\begin{tabular}{lllll}
\hline $\begin{array}{l}\text { Testing } \\
\text { data }(\%)\end{array}$ & $\begin{array}{l}\text { Accuracy (\%) } \\
\text { of lung cancer }\end{array}$ & $\begin{array}{l}\text { Accuracy (\%) } \\
\text { of liver cancer }\end{array}$ & $\begin{array}{l}\text { Accuracy (\%) of } \\
\text { breast cancer }\end{array}$ & $\begin{array}{l}\text { Average accuracy } \\
\text { (\%) of all cancer }\end{array}$ \\
\hline 10 & 99 & 98.23 & 96.47 & 97.9 \\
20 & 100 & 99.17 & 98.35 & 99.17 \\
30 & 98.03 & 90.39 & 97.39 & 95.27 \\
40 & 98.52 & 90.88 & 95 & 94.8 \\
50 & 89.41 & 84.11 & 94.11 & 89.21 \\
60 & 99.01 & 92.35 & 94.5 & 95.28 \\
70 & 99.15 & 84.78 & 92.26 & 92.06 \\
80 & 91.04 & 91.61 & 92.79 & 91.81 \\
90 & 95.42 & 81.89 & 91.89 & 89.73 \\
\multicolumn{7}{l}{ Total average } \\
\hline
\end{tabular}

Table 5 and Figure 6 show that the hybrid CNN-SVM accuracy results on all cancer dataset images are a feasible predict models, with average of running time that $<10 \mathrm{~s}$. The result of the highest accuracy value of hybrid CNN-SVM program trial is $100 \%$ on $20 \%$ testing data of lung cancer followed by $99.17 \%$ on $20 \%$ testing data of liver cancer and $98.35 \%$ on $20 \%$ testing data of breast cancer. Furthermore, based on the trials model's program, with total average of $86.2 \%$, the highest average accuracy value for all the cancer dataset images is $99.17 \%$ on $20 \%$ proportion of the testing data. As being shown, hybrid CNN-SVM is a proper and feasible model with competence of total highest average of $99.17 \%$ accuracy value.

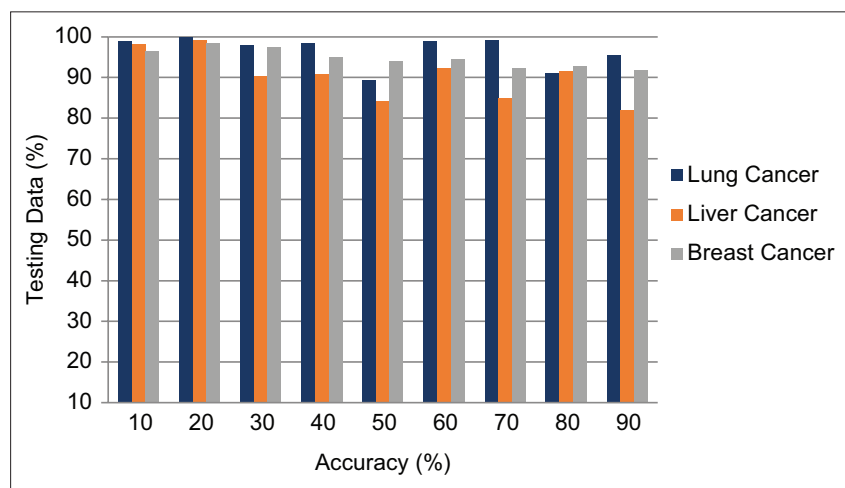

Figure 6: Graph of hybrid convolutional neural networks-support vector machines accuracy's performance on cancer dataset

\section{Discussion and Conclusion}

The writing research of aim to support health sector as prevent and focusing on better effectiveness of diagnose cancer diseases is adequately and strategically good proposed. The proposed method used of hybrid CNN-SVM and data materials from a search on the site cancerimagingarchive.net that obtained total of 900 data in the 2019-2021 of time range was correctly predict the data. The analysis is carried out using the proposed method by reviewing, identifying, and presenting it as the results obtained that machine learning has an influence on the classification of cancer. Based on the trials model's program, the highest accuracy value of hybrid CNN-SVM is $100 \%$ on $20 \%$ testing data of lung cancer followed with $99.17 \%$ on $20 \%$ testing data of liver cancer and $98.35 \%$ on $20 \%$ testing data of breast cancer. In addition, with total average of $86.2 \%$, the highest average accuracy value for all the cancer dataset images is $99.17 \%$ on $20 \%$ proportion of the testing data. Thereof, the combining model of hybrid CNN-SVM has the best performance with $99.17 \%$ accuracy value. The proposed method can detect cancer cells with an effective mechanism of action so can has the potential to inhibit in the future researches with more extensive data materials and various ailments.

\section{Acknowledgments}

This research was financially supported by the University of Indonesia through the FMIPA HIBAH 2021 research grant scheme. The authors also want to thank cancer imaging archive for their assistance in providing the research data publicly.

\section{References}

1. Miller KD, Fidler-Benaoudia M, Keegan TH, Hipp HS, Jemal A, Siegel RL. Cancer statistics for adolescents and young adults. A Cancer J Clin. 2020;70(6):443-59. http://doi.org/10.3322/ caac. 21637 PMid:32940362

2. Yang L, Shi P, Zhao G. Targeting cancer stem cell pathways for cancer therapy. Signal Transduct Target Ther. 2020;5(1):8. http://doi.org/10.1038/s41392-020-0110-5 PMid:32296030

3. Leone RD, Powell JD. Metabolism of immune cells in cancer Nat Rev Cancer. 2020;20:516-31. http://doi.org/10.1038/ s41568-020-0273-y

PMid:32632251

4. Hegde PS, Chen DS. Top 10 challenges in cancer immunotherapy. Immunity. 2020;52(1):17-35. http://doi. org/10.1016/j.immuni.2019.12.011 PMid:31940268

5. Japanese Gastric Cancer Association. Japanese Gastric Cancer Treatment Guidelines 2018. Gastric Cancer. $5^{\text {th }}$ ed., Vol. 24. Japanese Gastric Cancer Association.; 2021. p. 1-21. 
http://doi.org/10.1007/s10120-020-01042-y.

6. Howlader N, Forjaz G, Mooradian MJ, Meza R, Kong CY Cronin KA, et al. The effect of advances in lung-cancer treatment on population mortality. N Engl J Med. 2020;383(7):640-9. http:// doi.org/10.1056/NEJMoa1916623

PMid:32786189

7. Bade BC, Cruz CSD. Lung cancer 2020 epidemiology, etiology, and prevention. Clin Chest Med. 2020;41(1):1-24. http://doi. org/10.1016/j.ccm.2019.10.001

PMid:32008623

8. Anwanwan D, Singh SK, Singh S, Saikam V, Singh R. Challenges in liver cancer and possible treatment approaches. Biochim Biophys Acta. 2020;1873(1):188314. http://doi.org/10.1016/j. bbcan.2019.188314

PMid:31682895

9. Kim YJ, Jang H, Lee K, Park S, Min S, Hong C, et al. PAIP 2019: Liver cancer segmentation challenge. Med Image Anal. 2021;67:101854.

10. Britt KL, Cuzick J, Phillips KA. Key steps for effective breast cancer prevention. Nat Rev Cancer. 2020;20:417-36. http://doi. org/10.1038/s41568-020-0266-x

PMid:32528185

11. Ezzati M, Yousefi B, Velaei K, Safa A. A review on anticancer properties of Quercetin in breast cancer. Life Sci. 2020;248:117463. http://doi.org/10.1016/j.Ifs.2020.117463 PMid:32097663

12. Badža MM, Barjaktarović MC. Classification of brain tumors from MRI images using a convolutional neural network. Appl Sci. 2020;10(6):1999. https://doi.org/10.3390/app10061999

13. Abbas A, Abdelsamea MM, Gaber MM. Classification of COVID19 in chest X-ray images using DeTraC deep convolutional neural network. Appl Intell. 2021;51:854-64. https://doi. org/10.1007/s10489-020-01829-7

14. Ali L, Wajahat I, Golilarz NA. LDA-GA-SVM: Improved hepatocellular carcinoma prediction through dimensionality reduction and genetically optimized support vector machine. Neural Comput Appl. 2021;33:2783-92. https://doi.org/10.1007/ s00521-020-05157-2.

15. Richhariya B, Tanveer M, Rashid AH. Diagnosis of Alzheimer's disease using universum support vector machine based recursive feature elimination (USVM-RFE). Biomedical Signal Processing and Control. 2020;59:101903. https://doi. org/10.1016/j.bspc.2020.101903

16. Tsenev V. A Methodology for Analysis of Thermal Transfer by Xray Study. $11^{\text {th }}$ National Conference with International Participation (ELECTRONICA); 2020. p. 1-4. https://doi. org/10.1109/ELECTRONICA50406.2020.9305137
17. Valueva MV, Nagornov NN, Lyakhov PA, Valuev GV, Chervyakov NI. Application of the residue number system to reduce hardware costs of the convolutional neural network implementation. Math Comput Simul. 2020;177:232-43. https:// doi.org/10.1016/j.matcom.2020.04.031

18. Yao $\mathrm{P}, \mathrm{Wu} \mathrm{H}, \mathrm{Gao} B$. Fully hardware-implemented memristor convolutional neural network. Nature. 2020;577:641-6. https:// doi.org/10.1038/s41586-020-1942-4

19. Tang S, Yuan S, Zhu Y. Convolutional neural network in intelligent fault diagnosis toward rotatory machinery. IEEE. 2020;8:86510-9. https://doi.org/10.1109/ACCESS.2020.2992692

20. Maeda-Gutiérrez V, Galván-Tejada CE, Zanella-Calzada LA, Celaya-Padilla JM, Galván-Tejada JI, Gamboa-Rosales H, et al. Comparison of convolutional neural network architectures for classification of tomato plant diseases. Appl Sci. 2020;10:1245. https://doi.org/10.3390/app10041245

21. Dhillon A, Verma GK. Convolutional neural network: A review of models, methodologies and applications to object detection. Prog Artif Intell. 2020;9:85-112. https://doi.org/10.1007/ s13748-019-00203-0

22. Pisner DA, Schnyer DM. Support vector machine. In Machine Learning. Ch. 6. Cambridge, Massachusetts: Academic Press; 2020. p. 101-21. https://doi.org/10.1016/ B978-0-12-815739-8.00006-7

23. Cervantes J, Garcia-Lamont F, Rodríguez-Mazahua L, Lopez A. A comprehensive survey on support vector machine classification: Applications, challenges and trends. Neurocomputing. 2020;408:189-215. https://doi.org/10.1016/j. neucom.2019.10.118

24. Sivaprakash M, Haribabu K, Sathish T, Dinesh S, Thermal VV. Support vector machine for modelling and simulation of heat exchangers. Science. 2020;24(1):499-503. https://doi. org/10.2298/TSCI190419398M

25. Raschka S. Python Machine Learning. Birmingham: eBook Packt Publishing; 2015.

26. Zhou J, Qiu Y, Zhu S, Armaghani DJ, Li C, Nguyen H, et al. Optimization of support vector machine through the use of metaheuristic algorithms in forecasting TBM advance rate. Eng Appl Artific Intell. 2021;97:104015. https://doi.org/10.1016/j. engappai.2020.104015

27. Tian Z. Short-term wind speed prediction based on LMD and improved FA optimized combined kernel function LSSVM. Eng Appl Artific Intell. 2020;91:103573. https://doi.org/10.1016/j. engappai.2020.103573

28. $\mathrm{Xu} \mathrm{J}$, Zhang $\mathrm{Y}$, Miao D. Three-way confusion matrix for classification: A measure driven view. Inform Sci. 2020;507:77294. https://doi.org/10.1016/j.ins.2019.06.064 